\title{
Niños y adolescentes con necesidades especiales de atención en salud: prevalencia hospitalaria y riesgos asociados
}

'Complejo Asistencial Dr. Sótero del Río, Servicio de Salud Metropolitano Sur Oriente, Santiago de Chile. División de Pediatría, Facultad de Medicina, Pontificia Universidad Católica de Chile, Santiago de Chile.

${ }^{3}$ Departamento de Salud Pública, Facultad de Medicina, Pontificia Universidad Católica de Chile, Santiago de Chile.

${ }^{4}$ Facultad de Medicina, Universidad de Chile, Sede Occidente, Santiago de Chile.

anterno de Medicina, Escuela de Medicina, Pontificia Universidad Católica de Chile, Santiago de Chile.

Recibido el 18 de marzo de 2011, aceptado el 15 de diciembre de 2011.

Correspondencia a: Juan Carlos Flores Cano. Complejo Asistencial

Dr. Sótero del Río, Servicio de Salud Metropolitano Sur Oriente, Concha y Toro 3459, Santiago, Chile. Teléfono: (56-2) 5764632. Celular: (56-9) 91389562. Fax: (56-2) 5764632. E-mail: jcflorescano@ gmail.com

\author{
JUAN CARLOS FLORES ${ }^{1}$, DANIELA CARRILLO², LORENA KARZULOVIC ${ }^{2}$, \\ JAIME CERDA ${ }^{2,3}$, GABRIELA ARAYA $^{\mathrm{a}}$, MARÍA SOLEDAD MATUS ${ }^{\mathrm{a}}$, \\ GUILLERMO LLÉVENES ${ }^{a}$, GONZALO MENCHACA ${ }^{1,2}$, NELSON A. VARGAS ${ }^{4}$
}

\section{Children with special health care needs: prevalence in a pediatric hospital and associated risks}

Background: "Children with special health care needs" (CSHCN) is a novel definition for pediatric patients with chronic diseases, adopted by the Chilean Pediatric Society in 2008. As life expectancy in Chile increases, prevalence of CSHCN is progressively growing, leading to higher health costs. Aim: To describe the epidemiological profile and clinical characteristics of hospitalized CSHCN, and compare the risk assessment of adverse events during hospitalization using two definitions for CSHCN. Patients and Methods: A cohort of hospitalized CSHCN in a Pediatric Center at Santiago, Chile, was followed from September to December 2009. Clinical and demographic data were registered in a database, including admission to intensive care unit (ICU), nosocomial infections and prolonged hospitalization ( $>7$ days). Incidence ratios for these events were compared between CSHCN and non-CSHCN, and between children attended by three or more health care professionals (CSHCN-3) and non-CSHCN. Results: Nine hundred twenty patients were included (54\% male), with a median age 14 months (0-221) and median days of hospitalization 4 days (1-229). Prevalence of CSHCN was $60.8 \%$ of hospitalized children. When using CSHCN definition and comparing with non-CSHCN, no excess of risk was documented. On the other hand, prevalence of CSHCN-3 was 19.9\%. Compared to non-CSHCN, these patients had a higher risk of ICU admission (relative risk $(R R)$ 1.58; 95\% confidence intervals $(C I)=1.22-2.05 ; p<0.01$ ), nosocomial infections $(R R$ 2.28; 95\% $C I=1.54-3.39 ; p<0.001)$ and prolonged hospitalization ( $R R$ 1.99; 95\% CI =1.52-2.60; $p<0.001)$. Conclusions: One in five hospitalized children met CSHCN-3 definition. These patients had an increased risk of adverse events during their hospitalization compared to non-CSHCN.

(Rev Med Chile 2012; 140: 458-465).

Key words: Chronic disease; Delivery of health care; Disability evaluation; Prevalence.
$\mathrm{E}$ 1 término "niños y adolescentes con necesidades especiales de atención en salud" (NANEAS) fue definido por primera vez el año 1998, en Estados Unidos de Norteamérica, como "todos aquellos que tienen o están en riesgo de presentar una enfermedad crónica de tipo físico, del desarrollo, conductual o emocional, y que además requieren mayor utilización de servicios en salud"1.

La Sociedad Chilena de Pediatría decide el año 2008 utilizar el término NANEAS en reemplazo de "enfermedades crónicas en la infancia", para enfatizar un manejo integral, centrado en la familia y a cargo de un equipo multidisciplinario ${ }^{2,3}$. 
En Chile se han producido cambios importantes en los indicadores biodemográficos en las últimas décadas. La mortalidad infantil ha llegado a cifras comparables con países desarrollados (7,8/1.000 nacidos vivos), y esta mayor sobrevida ha aumentado el número y complejidad de los pacientes con enfermedades crónicas ${ }^{4,5}$.

Por otra parte, el aumento de la esperanza de vida de estos niños determina que muchos de ellos alcancen la adultez. En promedio, 90\% de los niños con enfermedades crónicas sobrevive al menos hasta los 20 años, siendo necesario traspasar su cuidado desde el pediatra al internista ${ }^{6,7}$. Esta situación probablemente también tendrá un impacto en cuanto a carga asistencial y uso de recursos en los servicios de adultos en un futuro cercano.

El último estudio que aporta datos epidemiológicos acerca de pacientes pediátricos hospitalizados y enfermedades crónicas fue publicado en Chile el año 1994 por Vargas et $\mathrm{al}^{8}$. Estas constituían $35,2 \%$ de los egresos hospitalarios, siendo las enfermedades respiratorias (20,7\%) el grupo más frecuente de diagnósticos entre los pacientes con enfermedades crónicas. Hasta la fecha no existen datos que describan y cuantifiquen la prevalencia de NANEAS en los hospitales de nuestro país.

Motivados por la falta de información y la importancia de este grupo emergente en Salud Pública, diseñamos un estudio para determinar su prevalencia y riesgos en el hospital. Los objetivos fueron describir las características clínicas y demográficas de los NANEAS, y evaluar la definición en cuanto a su capacidad para determinar mayor riesgo de complicaciones durante la hospitalización. Analizamos el grupo de pacientes que cumplía con el concepto habitual, y otro que denominamos NANEAS-3: pacientes más complejos, en seguimiento por 3 o más profesionales de la salud. Para ambos se calculó riesgo relativo, en relación al grupo "No NANEAS", de ingreso a unidad de paciente crítico (UPC), infección asociada a atención de salud (IAAS) y hospitalización prolongada (> 7 días).

\section{Materiales y Métodos}

\section{Tipo de estudio}

De cohorte concurrente, aprobado por el Comité de Ética del Servicio de Salud Metropolitano Sur Oriente.

\section{Criterios de inclusión}

Se registraron los datos de todos los hospitalizados entre el 1 de septiembre y 31 de diciembre de 2009 en sala de Pediatría en el Complejo Asistencial Dr. Sótero del Río de Santiago, Región Metropolitana, Chile. Para el análisis, se consideró sólo la primera hospitalización en aquellos con más de una estadía.

\section{Pacientes NANEAS}

Se utilizó la definición descrita por McPherson et $\mathrm{al}^{1}$ en 1998, que ha sido aplicada en estudios de prevalencia en Estados Unidos de Norteamérica a través de una encuesta de tamizaje (screening), publicada por Bethel et $\mathrm{al}^{9,10}$ en 2002. En base a esto, se definió como NANEAS a aquellos pacientes que, previo a su ingreso, cumplían con al menos 1 de los siguientes criterios por un período de tiempo estimado de 12 meses:

- Necesidad actual de control médico con especialista, servicios de salud mental o psicopedagógicos.

- Necesidad de uso de medicamentos, distintos de vitaminas.

- Discapacidad física o mental producto de una enfermedad médica y que requiera derivación a especialista.

- Necesidad de uso de tecnología médica (ventilación mecánica, sonda o catéter para alimentación enteral o parenteral, catéter o derivación de vía urinaria o digestiva, y catéter de derivación de ventrículos cerebrales).

- Necesidad de terapia de rehabilitación física, de lenguaje o de deglución, derivada de una enfermedad médica.

Cada uno de los diagnósticos de patologías crónicas se conversó con los especialistas involucrados para confirmar que requerían seguimiento prolongado.

\section{Comparación de 2 definiciones}

Planteamos como hipótesis que la definición NANEAS no identifica al grupo de mayor riesgo de complicaciones en el hospital, por lo inclusivo del término. Para testearla, se definió como NANEAS-3 a aquellos que previo a su ingreso al hospital debían estar en seguimiento con 3 o más profesionales de la salud, por al menos 12 meses. Luego se compararon los riesgo relativos de ingreso a UPC, IAAS y hospitalización prolongada 
entre pacientes NANEAS y "No NANEAS", y entre NANEAS-3 y "No NANEAS".

\section{Variables biodemográficas}

De las historias clínicas, se registró: edad, género, diagnósticos de egreso, comorbilidades, días de hospitalización, ingreso a UPC, IAAS y hospitalización prolongada ( $>7$ días).

\section{Definiciones clínicas}

Para categorizar los diagnósticos de egreso, se utilizó la Clasificación Internacional de Enfermedades OMS, décima revisión (CIE-10 ${ }^{11}$.

Se definió síndrome bronquial obstructivo recurrente (SBOR) como 3 o más episodios de sibilancias en un año, y asma del lactante cuando se cumplía con el criterio del "asthma predictor index". (Castro-Rodríguez et $\mathrm{al}^{12}$ ).

Las IAAS fueron definidas como un cuadro infeccioso respiratorio, gastrointestinal, de sistema nervioso central, urinario o vascular, asociado a la hospitalización o procedimientos invasivos, que apareció después de 48 horas desde el ingreso, o antes de 7 días después del alta hospitalaria. Los métodos diagnósticos fueron los habituales empleados en la práctica clínica (panel viral, test pack rotavirus, cultivos de sangre, orina, LCR o deposiciones).

\section{Centro hospitalario}

El Complejo Asistencial Dr. Sótero del Río está en la zona Sur Oriente de Santiago, Región Metropolitana, y atiende una población estimada de 1.149.858 habitantes al año 2010, incluyendo 255.183 menores de 15 años $^{13}$. Cuenta con 52 camas pediátricas de cuidado básico, desde donde egresan anualmente alrededor de 4.000 pacientes (3.909 el año 2000, y 4.047 el año 2009) ${ }^{14}$.

\section{Análisis estadístico}

Las variables numéricas se presentan como mediana y rango (mínimo-máximo), y las categóricas como frecuencia y porcentaje.

Se utilizó el Test Mann Withney para comparar variables numéricas (distribución no normal), y el Test de $\chi^{2}$ para las categóricas.

El riesgo relativo de ingreso a UPC, IAAS y hospitalización prolongada ( $>7$ días) se calculó en base a un cuociente entre la incidencia de todas estas variables en pacientes: i) NANEAS/“NoNANEAS", y ii) NANEAS-3 / "No NANEAS".

Se consideró un valor-p $<0,05$ como estadísticamente significativo.
El análisis fue realizado con el programa estadístico "Statistical Package for the Social Sciences" (SPSS), versión 15.0.

\section{Resultados}

\section{Epidemiología}

Se obtuvo información de 1.023 egresos, correspondientes a 920 pacientes pediátricos (primera hospitalización). De ellos, 559 (60,8\%) cumplían la definición NANEAS y 183 (19,9\%) correspondían al grupo NANEAS-3.

Del total de hospitalizados, $54,3 \%$ eran varones, la mediana de edad fue de 14 meses (rango: 0-221 meses), la mediana de días de hospitalización fue de 4 días (rango: 1-229 días), 25,6\% ingresó a UPC, $12,7 \%$ presentó una IAAS, y 23,5\% presentó hospitalización prolongada.

Se registró un fallecido en sala de Pediatría durante el estudio por un síndrome diarreico agudo en paciente portador de ileostomía (IAAS).

\section{Motivos de ingreso al hospital}

Del total, la gran mayoría de los diagnósticos correspondió a causas respiratorias $(71,2 \%)$, con menor frecuencia de infecciosas $(6,9 \%)$, genitourinarias $(5,7 \%)$, externas $(2,5 \%)$, y el conjunto de endocrinológicas, nutricionales y del metabolismo $(1,9 \%)$.

\section{Comparación de los grupos NANEAS y NANEAS-3 con los pacientes "No NANEAS"}

Las características de los pacientes se muestran en las Tablas 1 y 2.

En el grupo NANEAS, la mediana de edad fue mayor que la del grupo "No NANEAS" ( 21 vs 6 meses, respectivamente; $\mathrm{p}<0,001)$. No hubo diferencia significativa en ninguna de las otras variables analizadas.

El grupo NANEAS-3 se diferencia del grupo "No NANEAS" en las variables edad (mediana de 21 vs 6 meses, respectivamente; $\mathrm{p}<0,001)$, y días de hospitalización (mediana de 6 vs 4 días, respectivamente; $\mathrm{p}<0,001)$. Además destaca una mayor frecuencia de ingreso a UPC, IAAS y hospitalización por más de 7 días $(\mathrm{p}<0,01)$. No se observó diferencia en la distribución por sexo.

La Figura 1 (a) compara el riesgo relativo de ingreso a UPC, IAAS y hospitalización prolongada entre los grupos NANEAS-3 y “No NANEAS”. Los 
NANEAS: prevalencia hospitalaria y riesgos asociados - J. C. Flores et al

Tabla 1. Características clínicas y demográficas de los pacientes NANEAS, comparados con los "No NANEAS", Servicio de Pediatría, Hospital Sótero del Río, septiembre-diciembre 2009

\begin{tabular}{|lllc|}
\hline Variables & NANEAS & No NANEAS & Valor - p \\
\hline $\mathrm{n}$ & 558 & 360 & \\
\hline${\text { Edad, } \text { meses }^{\S}}^{\text {Sexo, masculino }}$ & $21,0(0-221)$ & $6,0(0-179)$ & $0,001^{*}$ \\
\hline Días hospitalización & $52,3 \%$ & $57,8 \%$ & 0,118 \\
\hline Ingreso UPC & $4,0(1-229)$ & $4,0(1-92)$ & 0,095 \\
\hline IAAS & $26,3 \%$ & $24,5 \%$ & 0,558 \\
\hline Hospitalización prolongada & $14,0 \%$ & $10,6 \%$ & 0,154 \\
\hline
\end{tabular}

*Estadísticamente significativo, $\mathrm{p}<0,05,{ }^{\S}$ Mediana (rango).

Tabla 2. Características clínicas y demográficas de los pacientes NANEAS-3, comparados con los "No NANEAS", Servicio de Pediatría, Hospital Sótero del Río, septiembre-diciembre 2009

\begin{tabular}{|lllc|}
\hline Variables & NANEAS-3 & No NANEAS & Valor-p \\
\hline $\mathrm{n}$ & 183 & 360 & \\
\hline Edad, meses $^{\S}$ & $21,0(0-221)$ & $6,0(0-179)$ & $0,001^{*}$ \\
\hline Sexo, masculino & $52,5 \%$ & $57,8 \%$ & 0,272 \\
\hline Días hospitalización & $6,0(1-229)$ & $4,0(1-92)$ & $<0,001^{*}$ \\
\hline Ingreso UPC & $38,8 \%$ & $24,5 \%$ & $<0,01^{*}$ \\
\hline IAAS & $24,2 \%$ & $10,6 \%$ & $<0,001^{*}$ \\
\hline Hospitalización prolongada & $41,0 \%$ & $20,6 \%$ & $<0,001^{*}$ \\
\hline
\end{tabular}

*Estadísticamente significativo, $\mathrm{p}<0,05,{ }^{\S}$ Mediana (rango).

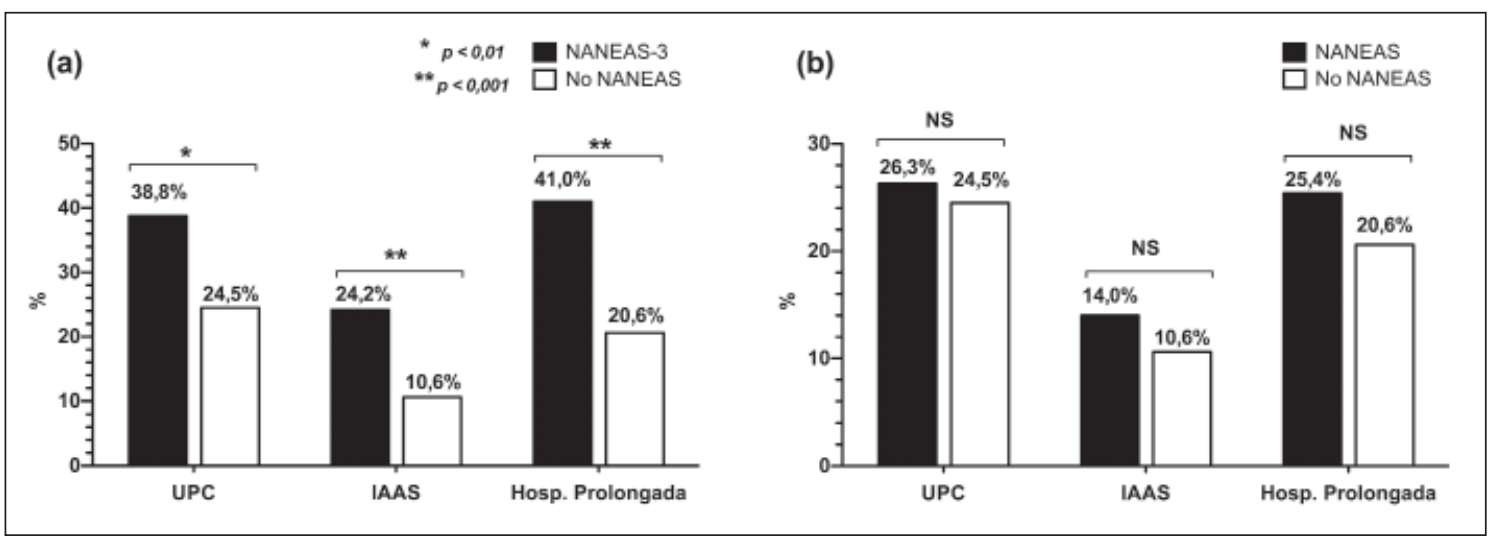

Figura 1 (a) y (b). Incidencia y comparación del riesgo relativo de ingreso a UPC, IAAS y hospitalización prolongada entre los grupos NANEAS-3 vs "No NANEAS", y NANEAS vs "No NANEAS", respectivamente. 


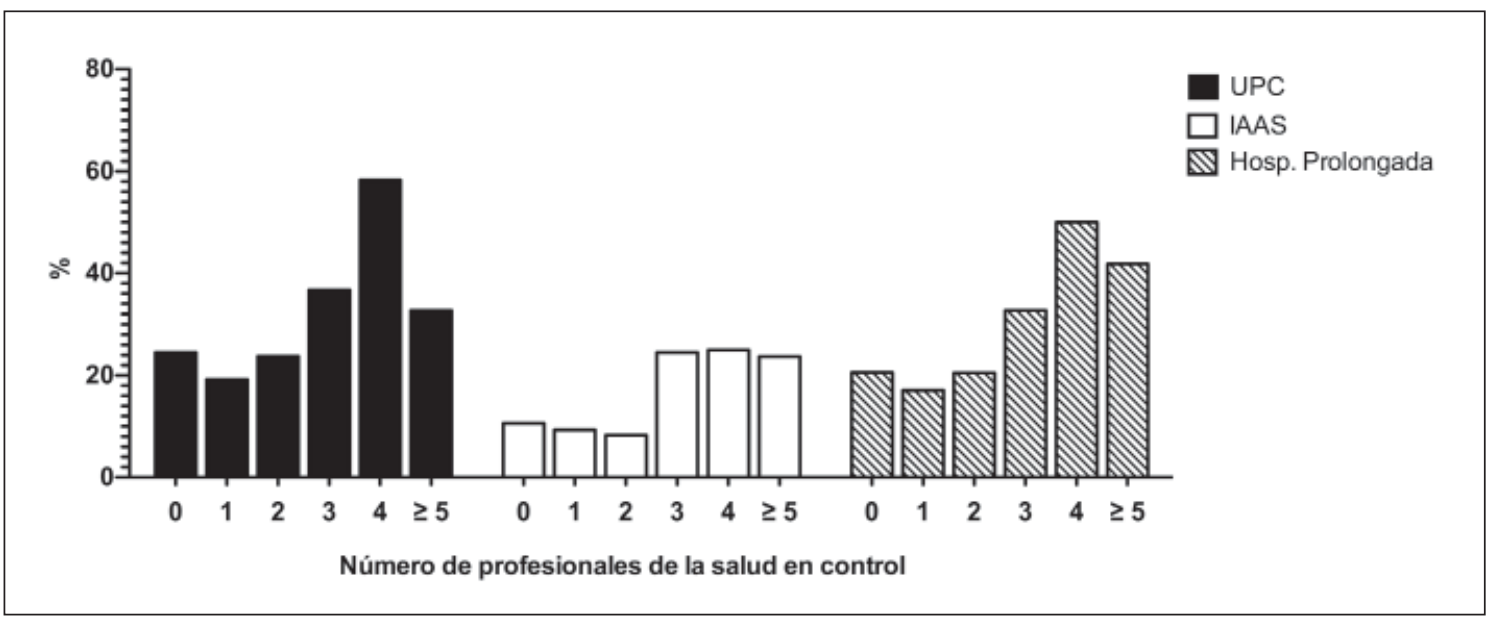

Figura 2. Incidencia de complicaciones según número de profesionales de la salud involucrados en seguimiento previo a su ingreso al hospital. Se observa en general una tendencia ascendente al aumentar la complejidad del paciente, sin embargo, para la variable ingreso a UPC existe una disminución importante cuando se trata de los pacientes controlados por 5 o más profesionales distintos. Esta situación se explica probablemente porque en este grupo se incluyen niños en cuidado paliativo, que no se manejan en Unidades Críticas pese a estar gravemente enfermos, como una medida de proporcionalidad terapéutica.

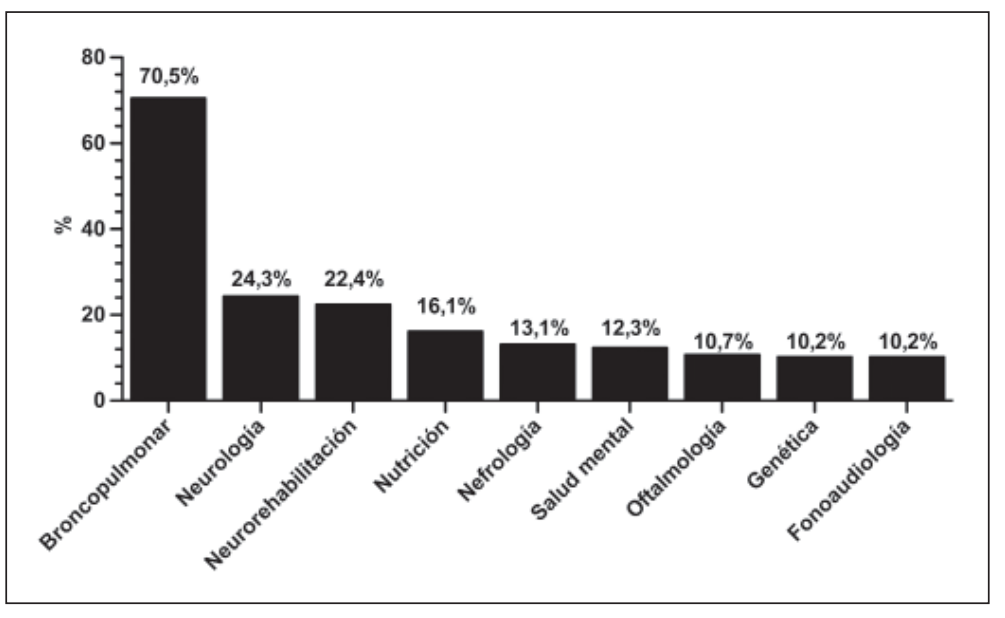

Figura 3. Seguimiento de pacientes NANEAS por distintos profesionales de la salud, previo a su ingreso al hospital. niños del grupo NANEAS-3 tuvieron 1,58 veces más riesgo de ingreso a UPC (IC 95\% = 1,22-2,05), 2,28 veces más riesgo de presentar IAAS durante su hospitalización (IC 95\% = 1,54-3,39) y 1,99 veces más riesgo de hospitalización prolongada (IC 95\% = 1,52-2,60), en comparación con los pacientes "No NANEAS".

La Figura 1 (b) compara el riesgo relativo de ingreso a UPC, IAAS y hospitalización prolongada entre los grupos NANEAS y "No NANEAS". Los niños del grupo NANEAS tuvieron 1,08 veces más riesgo de ingreso a UPC (IC 95\% = 0,86-1,35),
1,33 veces más riesgo de presentar IAAS durante su hospitalización (IC 95\% = 0,92-1,91) y 1,24 veces más riesgo de hospitalización prolongada (IC 95\% = 0,96-1,58), en comparación con los pacientes "No NANEAS".

En la Figura 2 se muestra la incidencia de las 3 complicaciones en relación al número de profesionales de la salud en control.

\section{Patologías crónicas}

La Figura 3 muestra los profesionales de la salud que mantenían NANEAS en control, pre- 
vio a su ingreso al hospital. La gran mayoría en el grupo NANEAS debe realizar seguimiento por broncopulmonar (70,5\%), seguido por neurología $(24,3 \%)$, neurorehabilitación $(22,4 \%)$ y nutrición $(16,1 \%)$. Los pacientes con patologías crónicas respiratorias, en su mayoría SBOR o asma, representaron $38,5 \%$ del total de egresos.

\section{Discusión}

Los pacientes con enfermedades crónicas constituyeron un grupo muy prevalente entre los hospitalizados en sala de Pediatría. Más de la mitad cumple la definición NANEAS, y casi $20 \%$ corresponde a pacientes complejos, que requieren seguimiento por 3 o más profesionales de la salud (NANEAS-3). Este último grupo son niños vulnerables y demandantes desde el punto de vista médico, con mayor riesgo de ingreso a UPC, IAAS y hospitalización prolongada.

Es importante destacar que nuestro estudio demuestra que la definición NANEAS no detecta los pacientes con riesgo aumentado de complicaciones en el hospital. Para todas las variables analizadas: ingreso a UPC, IAAS y hospitalización prolongada, el intervalo de confianza del riesgo relativo incluye el valor 1 , lo cual significa que pertenecer al grupo NANEAS no representa una condición de mayor vulnerabilidad. En contraste, el grupo definido como NANEAS-3 presenta riesgo aumentado, estadísticamente significativo, para todas las complicaciones estudiadas.

Existe muy poca información en Chile que evidencie el cambio epidemiológico producido con los pacientes NANEAS como grupo emergente en Pediatría. Estos niños actualmente llegan a la adultez, situación que probablemente tendrá un impacto importante en cuanto a carga asistencial y uso de recursos en salud también en servicios de Medicina Interna ${ }^{6,7}$.

Debido al importante cambio en la definición con respecto al paciente con enfermedad crónica, es difícil hacer comparaciones con los estudios publicados anteriormente y sacar conclusiones certeras acerca del tema. El término NANEAS es inclusivo, y se enfoca esencialmente desde la utilización de los recursos y sistemas de salud.

Los estudios de Vargas et $\mathrm{al}^{8}$ y Cabezas et $\mathrm{al}^{15}$ estudiaron la prevalencia de enfermedad crónica pediátrica intrahospitalaria hace 19 y 17 años, respectivamente. Ambos se basaron en la Clasificación Internacional de Enfermedades OMS, novena revisión (CIE-9). El primer estudio mostró que $35,2 \%$ de 469 egresos hospitalarios, en un período de 6 meses, correspondía a una enfermedad crónica; los diagnósticos más frecuentes fueron: enfermedades respiratorias (20,7\%), anomalías congénitas $(15,6 \%)$ y enfermedades genitourinarias $(14,2 \%)$. El segundo estudio analizó la enfermedad respiratoria crónica o recurrente, encontrando que constituía $21,2 \%$ de las 1.516 hospitalizaciones de causa respiratoria, y 10,8\% del total de ingresos a Pediatría durante 1 año. Estos niños además tenían una hospitalización más larga (11,39 versus 8,8 días) que el total de los ingresos pediátricos en ese año, destacando una hospitalización muy prolongada ( $\geq 20$ días) en 24 casos, todos con una enfermedad no respiratoria de base, principalmente patología neurológica y reflujo gastroesofágico. Por último, hubo $33 \mathrm{pa}-$ cientes $(21,7 \%)$ con hospitalización recurrente (3 o más episodios en un año), de los cuales $74,2 \%$ tenía una enfermedad no respiratoria asociada.

Si bien nuestra definición del paciente con enfermedad crónica es distinta a la utilizada por ambos estudios mencionados, la prevalencia de pacientes NANEAS $(60,8 \%)$ constituye un aumento importante con respecto a lo reportado por Vargas et al $(35,2 \%)^{8}$. Parte de ese aumento se podría explicar por lo inclusivo de la nueva definición, pero probablemente no en su totalidad. Con respecto a los diagnósticos más prevalentes, se mantiene la patología pulmonar en el primer lugar. En nuestro estudio aparecen a continuación las enfermedades neurológicas y nutricionales, que adquieren mayor importancia.

En comparación a los datos reportados por Cabezas et $\mathrm{al}^{15}$, en nuestro estudio se constata un aumento muy importante de la patología respiratoria crónica o recurrente, concordante con los datos que han demostrado un aumento en la prevalencia de asma en Chile y los países occidentales ${ }^{16,17}$. En el estudio de Cabezas et $\mathrm{al}^{15}$, estos diagnósticos constituían un $11,1 \%$ del total de ingresos en 1 año, en comparación con 38,5\% encontrado por nosotros entre septiembre y diciembre. Nuestro dato probablemente está subestimado por el período primaveral del año analizado, debido a que es más probable que un paciente con enfermedad crónica respiratoria se hospitalice en invierno. 
Con respecto a los tiempos de estadía hospitalaria y hospitalizaciones recurrentes, los datos del estudio de Cabezas et $\mathrm{al}^{15}$ muestran resultados en la misma orientación que los nuestros, reforzando el concepto de que pacientes con más comorbilidades tienden a presentar más complicaciones.

Nuestro estudio obtuvo información de un importante número de pacientes hospitalizados, de comunas del sector Sur Oriente de Santiago, cuya población es de nivel socioeconómico medio y bajo. Esta situación probablemente genera un sesgo de selección, con una sobreestimación de la prevalencia de NANEAS en el hospital, por patologías crónicas de mayor gravedad o peor control.

Los NANEAS demandan gran cantidad de recursos en salud. Existe mucha literatura que promueve su manejo hospitalario en domicilio, con la coordinación de un equipo de salud reconocible para la familia, que dirija un cuidado integral, con controles con especialistas, rehabilitación y cumplimiento de los programas de inmunizaciones ${ }^{18,19,20,21}$. Además, un estudio reciente sugiere que manejar a estos pacientes en centros ambulatorios especializados adosados al hospital, con equipos multidisciplinarios bien coordinados, podría acortar los tiempos de estadía hospitalaria ${ }^{22}$.

Es indispensable generar más información actualizada acerca de la epidemiología de los NANEAS, y considerarlos en la planificación de políticas sanitarias destinadas a optimizar su manejo. Es necesario adaptar y fortalecer la red ambulatoria para una atención multidisciplinaria que mejore el cuidado y evite la hospitalización. $\mathrm{Si}$ esto no es posible, el manejo hospitalario debiera ser idealmente en domicilio, o en unidades especiales, con énfasis en el manejo de enfermería y un cumplimiento estricto de las normas de IAAS para evitar las complicaciones asociadas.

En conclusión, existe evidencia indirecta de un aumento de la prevalencia de NANEAS hospitalizados durante los últimos años, la cual en un futuro cercano se traspasará al grupo de adultos. Estos pacientes se han convertido en un grupo prevalente, vulnerable y demandante entre los hospitalizados. Los pacientes de mayor complejidad (NANEAS-3) tienen riesgo aumentado de complicaciones (ingreso a UPC, IAAS y hospitalización prolongada). La definición NANEAS entendida en su forma amplia e inclusiva, no es capaz de detectar al grupo de alto riesgo.
Agradecimientos: Los autores agradecen a Macarena Lizama Calvo, Ernesto Guiraldes Camerati, Alejandro Martínez Aguayo y Juan Carlos Flores Henríquez, por la gentil revisión de este manuscrito.

\section{Referencias}

1. Mc Pherson M, Arango P, Fox H, Lauver C, McManus M, Newacheck PW, et al. A new definition of children with special health care needs. Pediatrics 1998; 102: 137-9.

2. Comité de NANEAS. Sociedad Chilena de Pediatría. Concepto de NANEAS. Disponible en: http://comitenaneas.sochipe.cl/principal.php. Consultado el 25 de abril de 2010.

3. Sociedad Chilena de Pediatría. Recomendaciones para el Cuidado de niños y adolescentes con necesidades especiales de atención de salud (NANEAS). Disponible en: http://comitenaneas.sochipe.cl/modulos.php?mod $=$ documentos $\& \mathrm{fn}=5970 \mathrm{ce} 332198 \mathrm{baece} 7 \mathrm{cde} 000 \mathrm{c} 7169 \mathrm{bf}$ 0\&cat=11. Consultado el 25 de abril de 2010.

4. Instituto Nacional de Estadísticas. Estadísticas vitales 2008. Disponible en: http://www.ine.cl/canales/ menu/publicaciones/calendario_de_publicaciones/ pdf/21_12_10/vit_08211210.pdf. Consultado el 15 de febrero de 2011.

5. Vargas N, Quezada A. Epidemiología, nueva morbilidad pediátrica y rol del pediatra. Rev Chil Pediatr 2007; 78 (Supl 1): 103-10.

6. American Academy of Pediatrics; American Academy of Family Physicians; American College of Physicians; American Society of Internal Medicine. A consensus statement on health care transitions for young adults with special health care needs. Pediatrics 2002; 110: 1304-6.

7. Blum R. Transition to adulth health care: setting the stage. J Adolesc Health 1995; 17: 3-5.

8. Vargas N, Arredondo O, Ilabaca G, Maturana A, Ortuvia G. Enfermedades crónicas en pacientes pediátricos: frecuencia y tipo de enfermedad. Rev Chil Pediatr 1994; 65: 264-7.

9. Bethell CD, Read D, Stein REK, Blumberg SJ, Wells N, Newacheck PW. Identifying children with special health care needs: development and evaluation of a short screening instrument. Ambulatory Pediatrics 2002; 2: 38-47.

10. Bethell CD, Read D, Blumberg SJ, Newacheck PW. What is the prevalence of children with special health care needs? Toward an understanding of variations in findings and methods across three national surveys. Matern Child Health J 2008; 12: 1-14.

11. Organización Mundial de la Salud. Clasificación Interna- 
cional de Enfermedades, décima revisión. Disponible en: http://www.who.int/classifications/icd/en/. Consultado el 20 de febrero de 2011.

12. Castro-Rodríguez JA, Holberg CJ, Wright AL, Martínez FD. A clinical index to define risk of asthma in young children with recurrent wheezing. Am J Respir Crit Care Med 2000; 162: 1403-6.

13. Instituto Nacional de Estadísticas. Proyecciones de población. Disponible en: http://www.ine.cl/canales/ chile_estadistico/demografia_y_vitales/demo_y_vita. php. Consultado el 15 de febrero de 2011.

14. Servicio de Salud Metropolitano Sur Oriente. Estadísticas. Disponible en: http://www.ssmso.cl/estadisticas/ estadisticas.htm. Consultado el 20 de febrero de 2011.

15. Cabezas P, Toro C, Boza ML. Enfermedades respiratorias crónicas y recurrentes de la infancia y la niñez en un hospital general. Rev Chil Pediatr 1997; 68: 175-81.

16. Valdivia G, Caussade S, Navarro H, Cerda J, Pérez E, Sánchez I, et al. Influencia del nivel socioeconómico (NSE) en el asma bronquial y cambios en su prevalencia en población escolar en un período de 6 años. Rev Med Chile 2009; 137: 215-25.

17. Eder W, Ege M, von Mutius E. The Asthma Epidemic. N
Engl J Med 2006; 355: 2226-35.

18. Homer C, Klatka K, Romm D, Kuhlthau K, Bloom S, Newacheck P, et al. A review of the evidence for the medical home for children with special health care needs. Pediatrics 2008; 122: e922-37.

19. Committee on Children with Disabilities. Care coordination: Integrating health and related systems of care for children with special health care needs. Pediatrics 1999; 104: 978-81.

20. Palfrey JS, Sofis LA, Davidson EJ, Liu J, Freeman L, Ganz ML. The pediatric alliance for coordinated care: Evaluation of a medical home model. Pediatrics 2004; 113: 1507-16.

21. Szilagyi PG, Shenkman E, Brach C, LaClair BJ, Swigonski $\mathrm{N}$, Dick A, et al. Children with special health care needs enrolled in the state children's health insurance program (SCHIP): Patient characteristics and health care needs. Pediatrics 2003; 112: e508-20.

22. Berman S, Rannie M, Moore L, Elias E, Dryer LJ, Jones MD Jr. Utilization and costs for children who have special health care needs and are enrolled in a hospitalbased comprehensive primary care clinic. Pediatrics 2005; 115: e637-42. 